\title{
Thoracoabdominal Aortic Aneurysm Open Repair: Visceral Arteries Reattachment Strategies
}

\author{
Luca Bertoglio, Alessandro Grandi, \\ Tommaso Cambiaghi, Cilli Giuseppe, \\ and Apruzzi Luca
}

\subsection{Introduction}

One of the crucial steps during thoracoabdominal aortic aneurysm open repair is the management of the visceral and renal vessels. Different techniques are available to obtain the same revascularization, but they vary in a few details and can have different indications and outcomes. Historically, the first proposed was the inclusion technique that consists in direct reimplantation of the origin of the celiac trunk, superior mesenteric artery, and renal arteries onto the graft [1].

However, associated atherosclerotic disease might complicate the reimplantation of the ostia of the visceral arteries. The presence of flowlimiting stenosis, calcifications, or thrombus apposition can increase the risk of reimplanted vessel occlusion and end-organ ischemia. Traditionally, in complex situations, open endarterectomy and graft bypass interposition were employed. Nowadays, different ancillary techniques have been proposed such as direct open stenting, sutureless anastomosis, and multibranched graft reimplantations. The aim of

L. Bertoglio $(\square) \cdot$ A. Grandi · T. Cambiaghi

C. Giuseppe · A. Luca

Division of Vascular Surgery, "Vita - Salute"

University, Scientific Institute H. San Raffaele,

Milan, Italy

e-mail: bertoglio.luca@hsr.it; alex@eenet.it;

cilli.giuseppe@hsr.it; apruzzi.luca@hsr.it this chapter is to illustrate the different techniques used today.

\subsection{Inclusion Techniques}

The most common revascularization technique employed is the inclusion technique: the anastomosis onto the surgical graft of a button of the aortic wall from which visceral vessels arise. This has the advantage of requiring only one anastomosis compared to single-artery reimplantation, thus reducing the end-organ damage and visceral ischemic time. The aortic patch can include a variable number of visceral vessels ostia so that sovrannumerary renal or splanchnic vessel can be reimplanted all together in case of anomalous vessel origin.

\subsubsection{Carrel Patch}

The first configuration used was the Carrel patch into which the origins of all the renal and splanchnic vessels are included. The major downside of this revascularization technique is the amount of pathologic aortic wall left that could be prone to further degeneration and subsequent further dilation into what is now referred to as visceral aortic patch (VAP) aneurysm [2]. Eventual management of VAP aneurysms is extremely challenging, with consequent high morbidity and mortality associated with redo open treatment $[3,4]$. 


\subsubsection{Undersized Patch (Three Vessels) and Left Renal Artery Direct Reimplantation: 3+1 Patch}

If the four ostia are too far away from each other, and to minimize the amount of aortic wall left in place, three (celiac trunk, superior mesenteric artery, and right renal artery) of the four vessels can be reimplanted with an aortic wall button/ island, and the remaining one, usually the left renal artery, can be reattached selectively with different techniques (Fig. 18.1).

\subsubsection{Direct Reimplantation}

Direct reimplantation of any visceral artery on the surgical graft is a feasible option to reduce the amount of aortic tissue used for the reimplantation (Fig. 18.2); however, it can be technically demanding when the original anatomy is distorted by the aneurysm and the available vessel length does not allow a reimplantation in the desired position.

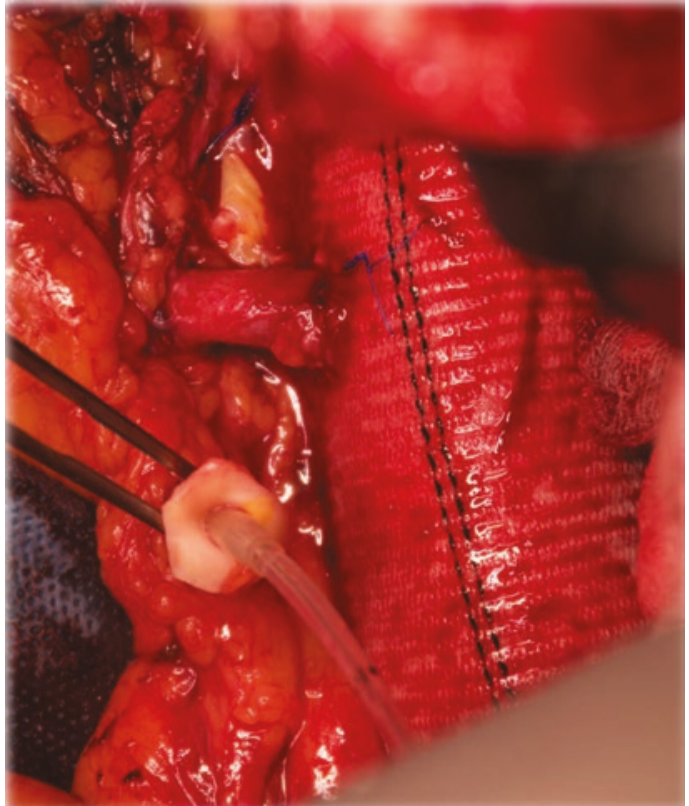

Fig. 18.2 Intraoperative capture of a direct reimplant of the superior mesenteric artery during a thoracoabdominal repair
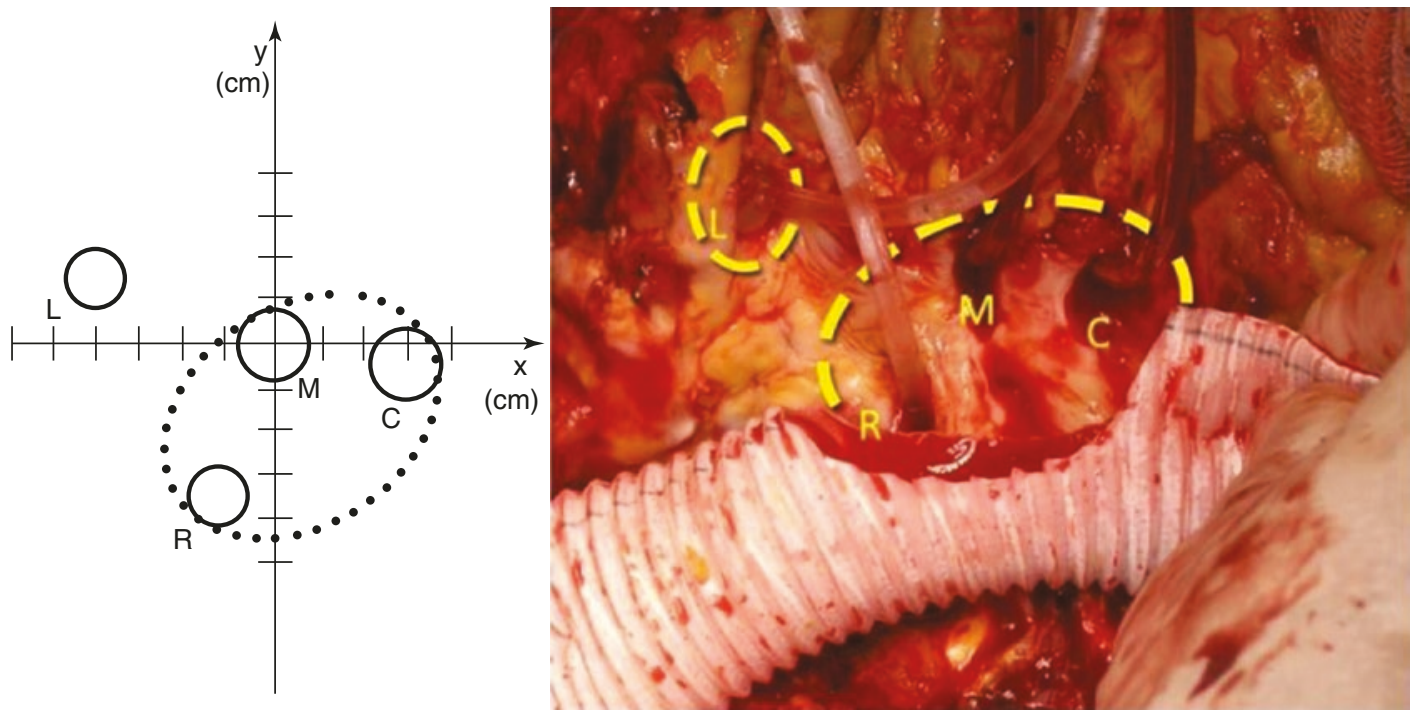

Fig. 18.1 Undersized patch to include three vessels and separately reimplant the left renal artery 


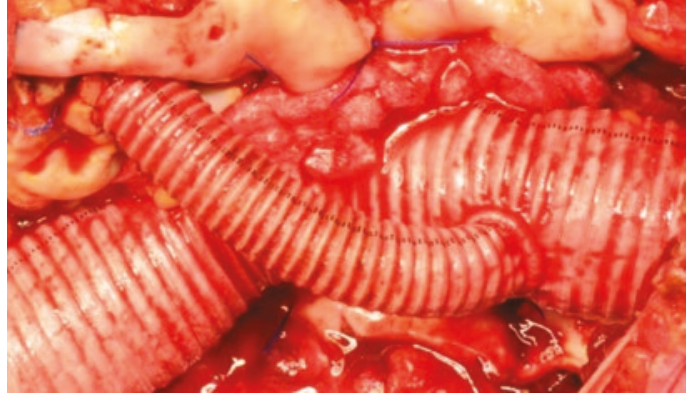

Fig. 18.3 Graft interposition to revascularize the left renal artery

\subsubsection{Surgical Graft Interposition}

In these cases, or when the origin of the target vessel is pathologic (stenotic, dissected, aneurysmatic), a small-diameter $(6-8 \mathrm{~mm})$ prosthetic graft can be used to perform a bypass in between the aortic surgical graft and the visceral vessel (Fig. 18.3). This technique also allows shortening of the vessel, kinking correction, and possible excision of the ostial aortic disease (stenosis or aneurysmatic dilation). The drawback of this technique is the possible kinking and occlusion of the graft and therefore of the vessel.

\subsubsection{Gore Hybrid Graft}

The Gore Hybrid is an ePTFE vascular graft, constructed with an extremity joint to a selfexpandable nitinol-covered stent, which allows the possibility of "sutureless" vascular anastomosis. A recent study by our group [5] described the first application of this graft for revascularization of visceral arteries in open TAAA repair. The advantage of this new graft lies in the requirement of an anastomosis only on the free end of the bypass, while the stented end can be fixed only with a couple of stitches as the stent holds the graft in place. The results show that this technique is feasible even in the most tortuous anatomies and allows a new time-saving strategy of reperfusion of target vessels (Fig. 18.4).

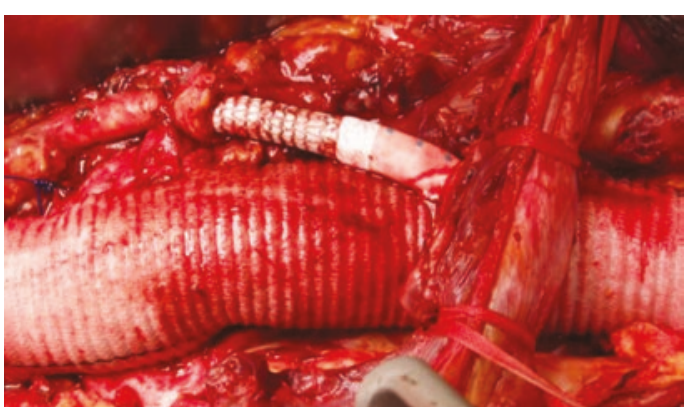

Fig. 18.4 Revascularization of the left renal artery with a sutureless graft

\subsubsection{Multibranched Graft}

If the ostia of the visceral and renal arteries are too far apart, or no diseased aortic wall is to be spared, single reimplantation of the target vessel can be performed directly on the graft. Youssef et al. reported a better outcome at 5 and 10 years for selective reimplantation compared to patch inclusion with a patency of $100 \%$ vs $94.2 \%$ and $100 \%$ vs $81.3 \%$, respectively [6]. Single-vessel revascularization reduces the amount of aortic tissue left, but it also allows to tailor the intraoperative strategy during the repair.

A four-branch synthetic graft can be used for extensive TAAA aneurysm where the surgeon needs to reattach individually the celiac trunk, the superior mesenteric artery, and the two renal arteries without leaving any aortic wall in place [7]. Single-/multivessel revascularization gives the ability to freely dictate whether or not to firstly revascularize the visceral vessels or perform the distal anastomosis.

Prioritization between visceral vessel revascularization and distal anastomosis can be done by using intraoperative motor and somatosensory evoked potentials to understand how the patient is reacting to the distal reperfusion and the aortic cross-clamping [8].

The use of this type of graft is ideal especially in two situations: when the patient is affected by a genetic collagenopathy such as Marfan, LoeysDietz, or Ehlers-Danlos syndromes, in these patients, indeed, any portion of native aortic tissue left in place is a potential site of further 
dilation, or when the origins of the visceral vessels are so distant that an inclusion technique is contraindicated (Fig. 18.5).

\subsection{Ancillary Techniques for Vessel Management}

\subsubsection{Ostial Endarterectomy}

Branch vessel stenosis in TAAA patents is most often related to atherosclerotic occlusive disease but can also be caused by aortic dissection. The visceral artery lesions are frequently proximal extensions of aortic atherosclerosis. In these cases, endarterectomy can be performed to remove the stenosis (Fig. 18.6). It is a feasible and safe technique that can be safely performed

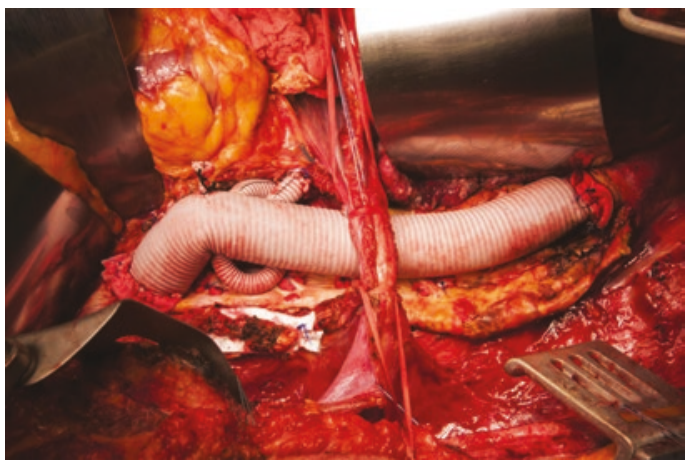

Fig. 18.5 Multibranched reimplantation of all the visceral arteries during a TAAA repair in a Marfan patient

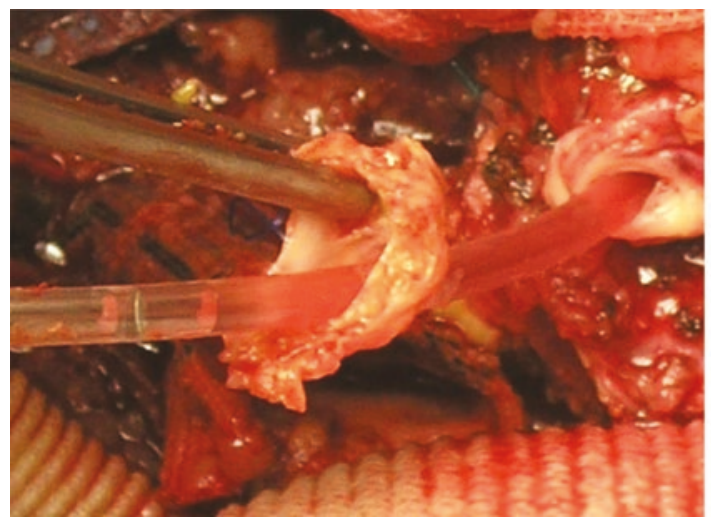

without exponentially increasing the operative time and yielding good results. However, it has important limitations, including the risks of vessel thrombosis related to an unsatisfactory endpoint and perforation of the thinned outer, fragile vessel wall $[9,10]$. The friable endarterectomized wall also increases the risk of vessel perforation during the insertion of balloon catheters that are commonly used to deliver renal perfusion. Bypasses to the branch vessels - the traditional alternative to endarterectomy-increase the endorgan ischemic times and may be associated with increased morbidity. The success of percutaneous visceral arterial stenting, combined with the associated technological advances, has created an opportunity to apply these techniques during open surgery [11].

\subsubsection{Direct Stenting}

Balloon expandable stents can be a useful adjunct for optimizing the visceral reconstruction during open TAAA repair. Direct stenting of ostial stenosis can greatly reduce operative time, and as reported by Coselli et al., patients who underwent visceral stenting had a low incidence of postoperative renal failure despite their high prevalence of preoperative renal insufficiency, renal occlusive disease, acute presentation, advanced age, and other risk factors [10].

Safe deployment requires that the stents are positioned precisely within the proximal portion

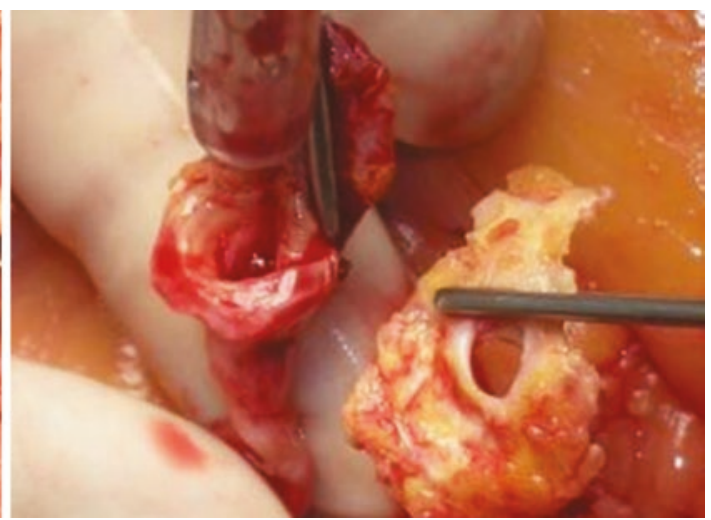

Fig. 18.6 Left renal artery endarterectomy before the reimplant to ease the suture and reimplant 

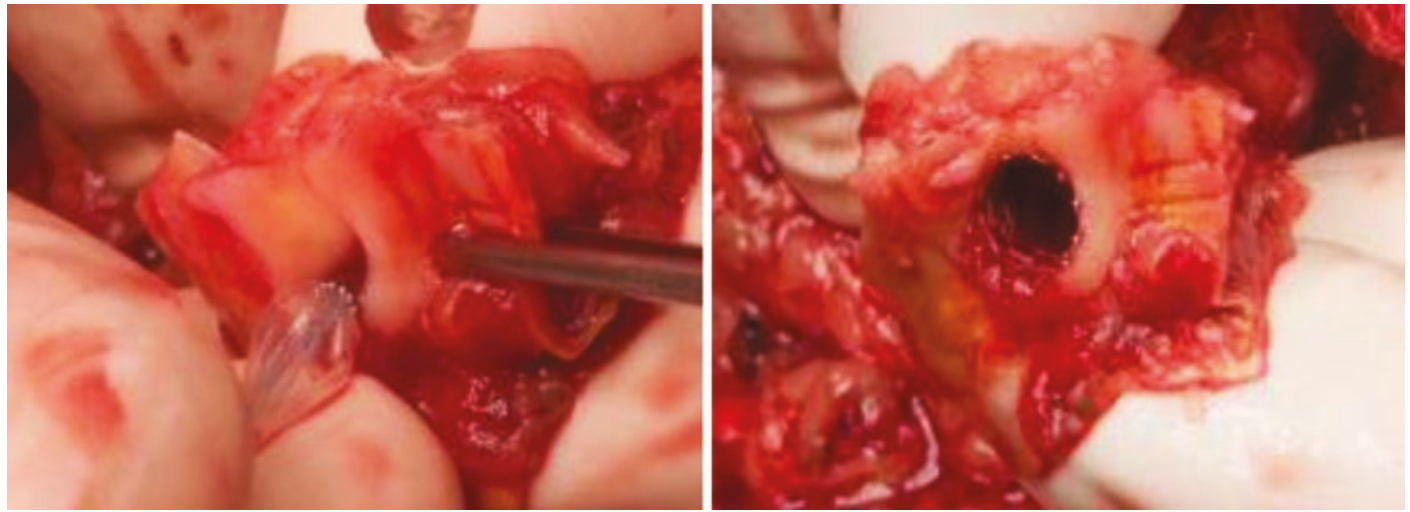

Fig. 18.7 Direct stenting of a stenotic renal artery before reimplant

of the vessel and expanded under direct visualization; insertion into a vessel lumen that is not well visualized should be strictly avoided (Fig. 18.7).

\section{References}

1. Coselli JS, LeMaire SA, Preventza O, de la Cruz KI, Cooley DA, Price MD, et al. Outcomes of 3309 thoracoabdominal aortic aneurysm repairs. J Thorac Cardiovasc Surg. 2016;151(5):1323-37. https://doi. org/10.1016/j.jtcvs.2015.12.050.

2. Dardik A, Perler BA, Roseborough GS, Williams GM. Aneurysmal expansion of the visceral patch after thoracoabdominal aortic replacement: an argument for limiting patch size? J Vasc Surg. 2001;34(3):4059. discussion 410.

3. Bertoglio L, Mascia D, Cambiaghi T, Kahlberg A, Melissano G, Chiesa R. Fenestrated and branched endovascular treatment of recurrent visceral aortic patch aneurysm after open thoracoabdominal repair. J Vasc Interv Radiol. 2018;29(1):72-77.e2. https://doi. org/10.1016/j.jvir.2017.08.012.

4. Bertoglio L, Mascia D, Cambiaghi T, Kahlberg A, Tshomba Y, Gomez JC, Melissano G, Chiesa R. Management of recurrent of visceral aortic patch aneurysms after thoracoabdominal repair: open, hybrid and endovascular approach. J Vasc Surg. 2018;67:41S-3S.

5. Chiesa R, Kahlberg A, Mascia D, Tshomba Y, Civilini E, Melissano G. Use of a novel hybrid vascular graft for sutureless revascularization of the renal arter- ies during open thoracoabdominal aortic aneurysm repair. J Vasc Surg. 2014;60(3):622-30. https://doi. org/10.1016/j.jvs.2014.03.256.

6. Youssef M, Neufang A, Jungmann F, Vahl CF, Dorweiler B. Patency of renal and visceral vessels after open thoracoabdominal aortic replacement. J Vasc Surg. 2015;62(3):594-9. https://doi. org/10.1016/j.jvs.2015.04.386.

7. de la Cruz K, LeMaire SA, Weldon SA, Coselli JS. Thoracoabdominal aortic aneurysm repair with a branched graft. Ann Cardiothorac Surg. 2012;1(3):381-93. https://doi.org/10.3978/j. issn.2225-319X.2012.08.05.

8. Keyhani K, Miller CC, Estrera AL, Wegryn T, Sheinbaum R, Safi HJ. Analysis of motor and somatosensory evoked potentials during thoracic and thoracoabdominal aortic aneurysm repair. J Vasc Surg. 2009;49(1):36-41. https://doi.org/10.1016/j. jvs.2008.08.005.

9. Stoney RJ, Messina LM, Goldstone J, Reilly LM. Renal endarterectomy through the transected aorta: a new technique for combined aortorenal atherosclerosis - a preliminary report. J Vasc Surg. 1989;9:224-33.

10. Clair DG, Belkin M, Whittemore AD, Mannick JA, Donaldson MC. Safety and efficacy of transaortic renal endarterectomy as an adjunct to aortic surgery. $\mathrm{J}$ Vasc Surg. 1995;21:926-34.

11. LeMaire SA, Jamison AL, Carter SA, Wen S, Alankar S, Coselli JS. Deployment of balloon expandable stents during open repair of thoracoabdominal aortic aneurysms: a new strategy for managing renal and mesenteric artery lesions. Eur J Cardiothorac Surg. 2004;26(3):599-607. https://doi.org/10.1016/j. ejcts.2004.04.035. 